\title{
DC Voltage Droop Control Design for MMC-Based Multiterminal HVDC Grids
}

\author{
Saman Dadjo Tavakoli (®), Enric Sánchez-Sánchez ${ }^{\circledR}$, Student Member, IEEE, \\ Eduardo Prieto-Araujo ${ }^{(0)}$, Member, IEEE, and Oriol Gomis-Bellmunt ${ }^{\left({ }^{\circ}\right.}$, Senior Member, IEEE
}

\begin{abstract}
This article addresses the design of the DC voltage droop control in modular multilevel converter (MMC)-based multiterminal HVDC grids. First, two energy-based control approaches, namely classic and cross control, are explored for the implementation of the voltage-power droop controller. The cross control, as the better solution for droop implementation, is further improved, making it more robust against disturbances. Then, a methodology is derived to select the droop gain combinations considering the AC grid, DC grid and MMC dynamics and their limitations. The methodology is based on a linear analysis to identify the valid droop gains which comply with the limits imposed on: the transient power sharing among MMCs, the DC grid voltage, the MMC AC and DC currents, the total MMC stored energy, and the stability margin of the complete multiterminal HVDC grid. Finally, time-domain simulations are conducted using the nonlinear model to validate the dynamic performance of the selected droop combinations obtained from the suggested methodology.
\end{abstract}

Index Terms-DC voltage, droop controller, MMC, multiterminal HVDC, singular value.

\section{INTRODUCTION}

$\mathbf{T}$ HANKS to their flexibility and controllablity in regulating power flows, high-voltage direct-current systems based on voltage-source converters (VSC-HVDC) are becoming a promising solution to interconnect power systems or integrate wind power plants into the mainland AC grid [1]-[3]. Several

Manuscript received July 31, 2019; revised October 31, 2019 and January 6 , 2020; accepted January 10, 2020. Date of publication January 20, 2020; date of current version September 23, 2020. This work was supported in part by the European Union's Horizon 2020 research and innovation programme under the Marie Sklodowska-Curie Grant 765585. This document reflects only the author's views; the European Commission is not responsible for any use that may be made of the information it contains, in part by FEDER/Ministerio de Ciencia Innovación y Universidades-Agencia Estatal de Investigación, under Project RTI2018-095429-B-I00, and in part by the ICREA Academia program. Paper no. TPWRD-00857-2019. (Corresponding author: Saman Dadjo Tavakoli.)

S. D. Tavakoli and E. Sánchez-Sánchez are with the Centre d'Innovació Tecnològica en Convertidors Estàtics i Accionaments, Departament d'Enginyeria Elèctrica, Universitat Politècnica de Catalunya, Barcelona 08028, Spain (e-mail: saman.dadjo@upc.edu; enric.sanchez.sanchez@upc.edu).

E. Prieto-Araujo is with the Centre d'Innovació Tecnològica en Convertidors Estàtics i Accionaments, Departament d'Enginyeria Elèctrica, Universitat Politècnica de Catalunya, Barcelona 08028, Spain, and also a Serra Húnter lecturer (e-mail: eduardo.prieto-araujo@citcea.upc.edu).

O. Gomis-Bellmunt is with the Centre d'Innovació Tecnològica en Convertidors Estàtics i Accionaments, Departament d'Enginyeria Elèctrica, Universitat Politècnica de Catalunya, Barcelona 08028, Spain, and also an ICREA Academia researcher (e-mail: oriol.gomis@upc.edu).

Color versions of one or more of the figures in this article are available online at https://ieeexplore.ieee.org.

Digital Object Identifier 10.1109/TPWRD.2020.2967881
VSC-HVDC links can be connected together, forming a multiterminal VSC-HVDC grid (VSC-MTDC) that offers additional benefits, such as enhanced reliability and optimized power flow across interconnected AC grids [4].

Although VSC-MTDC offers several advantages, the challenge of DC voltage control in VSC-MTDC grids is still under investigation. The strategies to control DC voltage can be categorized as: master-slave control, voltage margin control, and voltage droop control [5], [6]. Among these strategies, the droop control, as a decentralized control system, demonstrates better performance in terms of fast DC voltage control and active power sharing [7].

The basic idea of a droop controller is to allow multiple VSCs to simultaneously regulate their DC voltages while a fair accuracy of power sharing among VSCs is maintained. Considering the cascaded control system of a VSC, the deviation of a variable (such as voltage, current, or power) is multiplied by a droop gain in order to generate the reference for the outer control loop. Then, the outer control loop provides reference for the inner control loop [8], [9]. In case of a two-level VSC, a common practice is to use the $\mathrm{DC}$ voltage deviation to produce reference for the outer power control loop (power-voltage droop) [1], which in turn, generates reference for the grid current control loop. However, in case of an MMC, the power-voltage droop can be used to produce either grid current reference (classic control), which is similar to two-level VSC, or additive current reference (cross control) [10]. In fact, MMC is able to transiently store energy within its arms, allowing a different power exchange between $\mathrm{AC}$ and DC sides.

The question then arises which control alternative (using power-voltage droop to generate either grid current or additive current) provides better DC voltage performance in MTDC application. In [10], using a power-voltage droop structure, the DC voltage dynamics in an MTDC grid are studied. The main contribution is the derivation of a detailed model of the system and the improvement of the DC voltage dynamics by tuning various energy management control strategies. In [11], the droop control is designed for a four-terminal HVDC grid while only the dynamics of the grid side converters (regulating DC voltage via droop controller) are considered and the wind farm converters (regulating active power) are modeled via current sources. With this simplification, the dynamics of the wind farm MMCs are not considered on the DC voltage dynamics. Clearly, a change in the wind farm AC power would cause a transient on the DC power of MMC, which in turns, impacts the dynamics of DC voltage. 
If the wind farm and the related MMC is replaced via a current source, ignoring the impact of the wind farm MMC dynamics on the DC power and hence on the DC voltage, then the DC voltage dynamics only rely on the dynamics of grid side MMCs and do not contain properly the dynamics of power. Furthermore, the droop control system is only implemented on the classic control and the cross structure is not studied.

This is also valid for studies carried out in [12]-[16]. While all of them deal with the droop control design in MTDC application, their models (linear or non-linear) do not include the dynamics of either DC grid, MMC energy control loops, or the entire dynamics of several MMCs. Moreover, none of them discusses an alternative control approach in which the droop controller is combined with cross control system. The cross control is an interesting candidate for droop control implementation since it reduces the coupling between the dynamics of the $\mathrm{AC}$ and $\mathrm{DC}$ sides of an MMC. As it is observed in [11] and [10], in case of the classic control system, the energy control loop of an MMC has negative impact on the dynamics of the DC voltage. Hence, it is of interest to evaluate the dynamic performance of classic and cross control systems while combined with droop controller.

The system dynamics are not only influenced by the MMC control structure. The droop gains also affect both the DC voltage dynamics and the power sharing accuracy [1], [17]. Hence, a methodology is required to find the most suitable droop gains while taking account of the system dynamic behaviour. It should also be applicable to the systems with any MMC control structure. In [1] and [8], the dynamic performance of the system for the given droop gains are evaluated by means of maximum singular values of DC voltage and AC current of converters. In this article, this approach is further extended to evaluate the dynamics of not only DC voltage and AC current, but also the MMC DC current, transient power sharing among MMCs, and total energy of MMC for the given droop gains.

To the best of the authors knowledge, the suitable control structures, capabilities and limitations of the MMC for DC droop voltage control applications have not been fully investigated. Within this context, the contributions of this paper are: (1) The dynamic performance of different energy-based MMC control structures for DC droop voltage control is studied and compared. (2) An improved MMC control structure is suggested for DC droop voltage regulation. (3) A methodology is suggested based on a linear analysis to identify the most suitable droop gains which comply with the limits defined for: transient power sharing among MMCs, DC grid voltage, MMC AC and DC currents, the total MMC stored energy, and the stability margin of the complete multiterminal HVDC grid. Once the droop gains are selected, a time-domain analysis is conducted on the non-linear model, validating the results obtained from linear analysis.

The rest of the paper is organised as follows. In Section II, an exemplary MMC-based VSC-MTDC grid is explained and the fundamentals of energy-based control system are briefly discussed. The different control system alternatives for droop implementation are detailed and an improved control system is suggested in Section III. In Section IV, the state-space equations for the MTDC grid and its components are derived as the basis for a linear analysis of the system. Based on the system linear model,

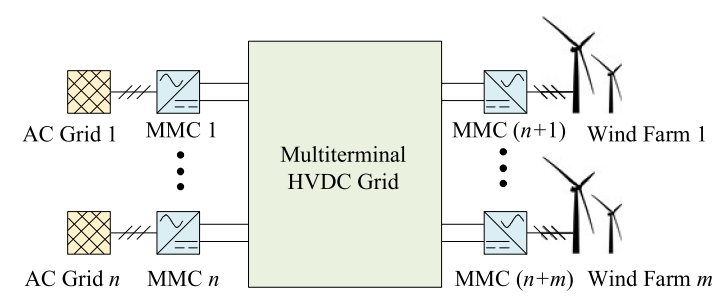

Fig. 1. An exemplary MMC-based multiterminal HVDC grid.

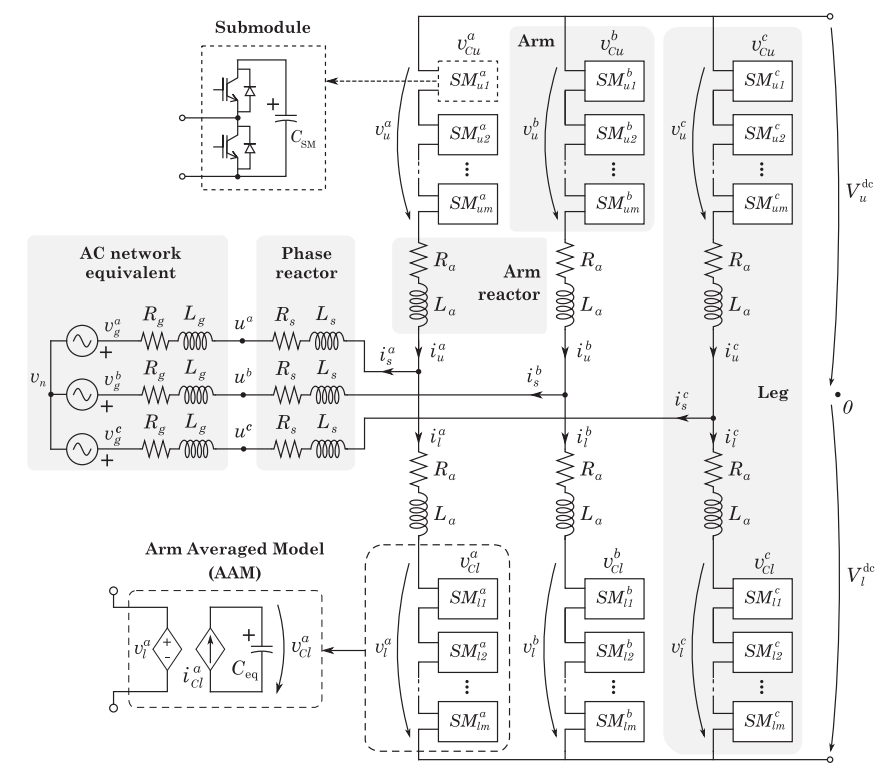

Fig. 2. Electrical circuit of an MMC connected to an AC grid.

a methodology for selecting suitable droop gains is presented in Section V. Finally, the methodology is applied to a four-terminal system in Section VI.

\section{SySTEM DESCRIPTION}

An exemplary VSC-MTDC grid is presented in Fig. 1. The system consists of $n$ AC grids and $m$ wind farms, which are connected to the MTDC grid via MMCs. A common practice is to have wind farm MMCs to regulate wind farm AC voltages, enabling wind farm power to be delivered to the MTDC grid. The DC voltage of the MTDC grid is regulated by the grid connected MMCs using DC voltage droop control [18]. The MTDC grid includes DC cables and can have any topology.

The topology of an MMC connected to an AC grid is shown in Fig. 2. It has six arms, each of them consisting of $N_{\text {arm }}$ half-bridge submodules with a capacitance $C_{S M}$ and a series arm reactor. The submodules (SM) can be controlled individually to either insert their capacitors in the circuit or bypass it. This allows each arm to act as a controllable voltage source fed from an aggregated capacitor. The voltages of the six arms can be controlled to achieve the desired power exchange between $\mathrm{DC}$ and $\mathrm{AC}$ grids. Moreover, the AC grid is presented by an equivalent network with a high short circuit ratio.

The Energy-based control system of an MMC is presented in Fig. 3. As detailed in [19], the control system consists of 


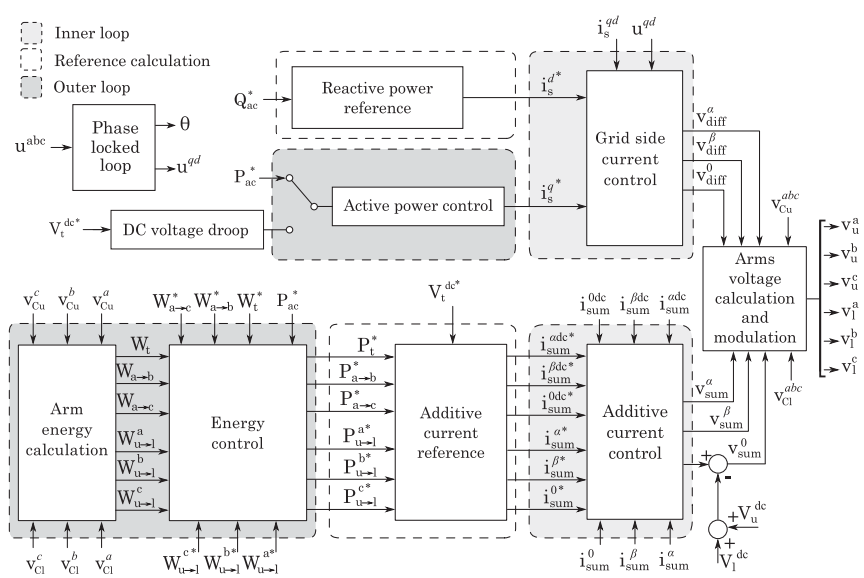

Fig. 3. Energy-based control system of an MMC

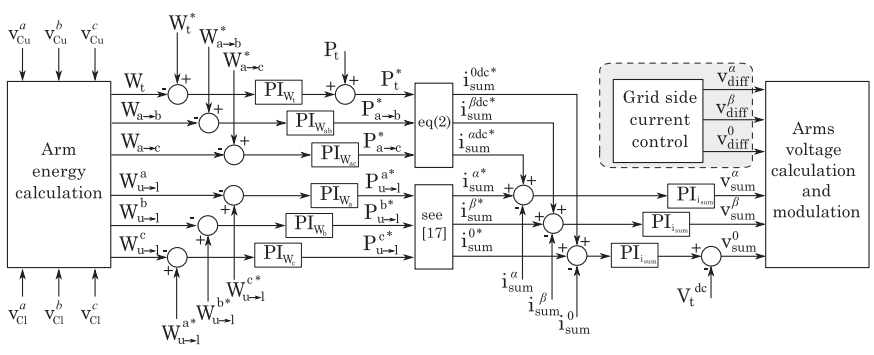

Fig. 4. Detailed energy control loops of an MMC.

two main cascaded control loops that regulate grid current and additive current. The grid current control loop operates in $q d$-frame and tracks the current references sent by the outer control loops. Based on the control objectives, DC voltage or AC power control loop provides $q$-axis current reference, while reactive power is used to derive $d$-axis current reference. The additive current control loop operates in $\alpha \beta$-frame and follows the references provided by the internal energy control loops.

The internal energy of an MMC is regulated via six control loops as shown in Fig. 4. The energy differences between two legs $\left(W_{a \rightarrow b}, W_{a \rightarrow c}\right)$ and the energy differences between upper and lower arms of three legs $\left(W_{u \rightarrow l}^{a}, W_{u \rightarrow l}^{b}, W_{u \rightarrow l}^{c}\right)$ are maintained to zero by five control loops. Additionally, one control loop is needed to maintain the total stored energy $\left(W_{t}\right)$ at the MMC rated energy level,

$$
W_{t}^{*}=6 \frac{1}{2} \frac{C_{S M}}{N_{\text {arm }}}\left(V_{t}^{\mathrm{dc} *}\right)^{2}
$$

The outputs of the energy control loops are DC power references, including the references for the DC power exchanges between two legs $\left(P_{a \rightarrow b}^{*}, P_{a \rightarrow c}^{*}\right)$ and the upper and lower arms $\left(P_{u \rightarrow l}^{a *}\right.$, $\left.P_{u \rightarrow l}^{b *}, P_{u \rightarrow l}^{c *}\right)$, and total DC power $\left(P_{t}^{*}\right)$. Next, the DC power references can be used to derive the DC and AC terms of the additive current reference $\left(i_{\mathrm{sum}}^{\alpha \beta 0 \mathrm{dc} *}\right.$ and $\left.i_{\mathrm{sum}}^{\alpha \beta 0 *}\right)$. The DC term of additive current is calculated by

$$
\left[\begin{array}{c}
i_{\mathrm{sum}}^{\alpha \mathrm{du}^{*}} \\
i_{\mathrm{sum}^{*}}^{\beta \mathrm{dum}^{*}} \\
i_{\mathrm{sum}}^{O \mathrm{scm}^{*}}
\end{array}\right]=\frac{1}{3 V_{t}^{\mathrm{dcc}^{*}}}\left[\begin{array}{ccc}
0 & 1 & 1 \\
0 & \sqrt{3} & -\sqrt{3} \\
1 & 0 & 0
\end{array}\right]\left[\begin{array}{c}
P_{t}^{*} \\
P_{a \rightarrow b}^{*} \\
P_{a \rightarrow c}^{*}
\end{array}\right]
$$

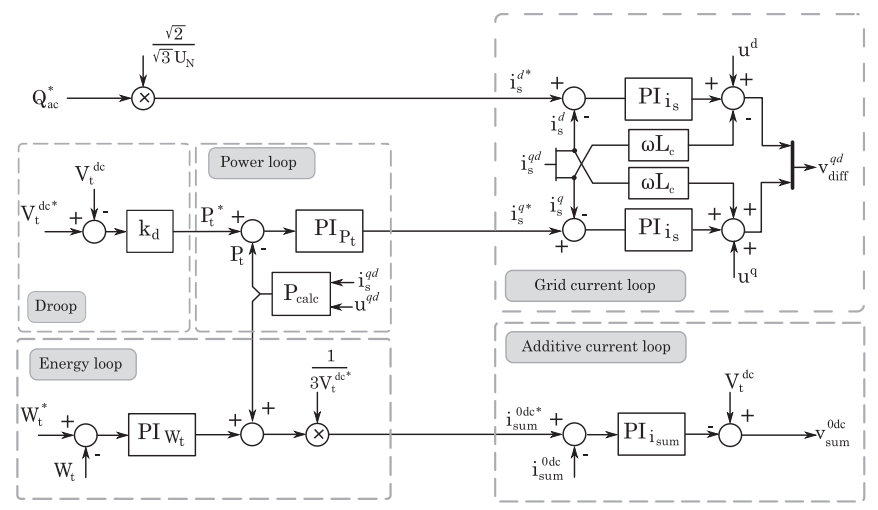

Fig. 5. MMC classic control structure.

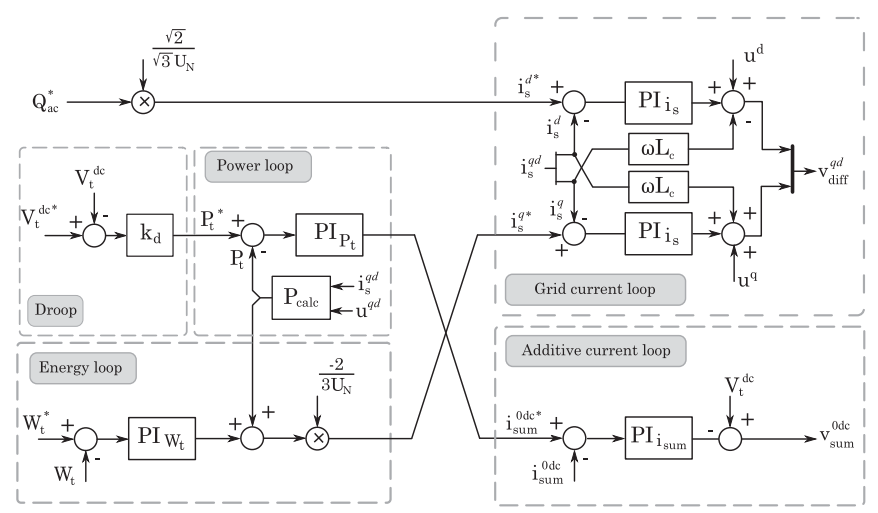

Fig. 6. MMC cross control structure.

The calculation of the AC terms is more complicated since it contains positive and negative sequence components. A detailed explanation on how to derive the AC terms of additive current and how to tune the PI controllers are provided in [19].

\section{MMC CONTROL SYSTEM ALTERNATIVES}

In this section, the classic and cross control approaches are explained and an improved version of the cross control is suggested.

\section{A. Classic and Cross Control Approaches}

As mentioned earlier, the power reference for the MMCs regulating DC voltage is calculated by multiplying the DC voltage deviation by droop gain $\left(k_{d}\right)$

$$
P_{t}^{*}=k_{d}\left(V_{t}^{d c *}-V_{t}^{d c}\right)
$$

In classic control approach (Fig. 5), the output of the power controller produces the grid current reference $\left(i_{s}^{q *}\right)$ while the total energy $\left(W_{t}\right)$ control loop generates the additive current reference $\left(i_{\mathrm{sum}}^{\mathrm{Odc}}\right)$. On the contrary, in case of cross control system, the power controller and total energy controller produce, respectively, $i_{\text {sum }}^{0 d c *}$ and $i_{s}^{q *}$ (Fig. 6). The MMCs that operate in power control mode directly receive power set points from a secondary control system. 


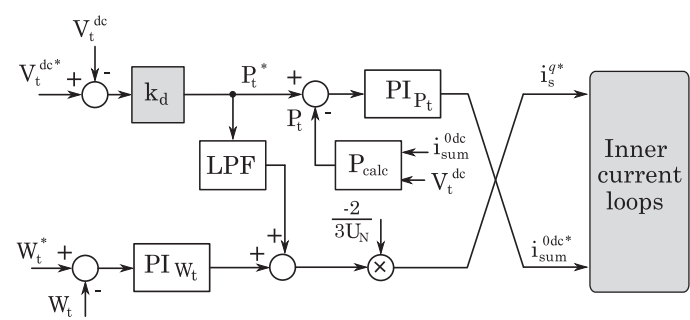

Fig. 7. Improved cross control approaches.

\section{B. Suggested Control Approach}

As it is concluded in [20], the cross control approach is more suitable for DC voltage regulation in a point-to-point HVDC link. However, it can be further improved making it more robust against DC and AC voltage disturbances. As it is presented in Fig. 7, instead of measured power, the reference power generated by droop controller can be feedforwarded to the total energy control loop. Hence, it would not be affected by the disturbances on the AC side. Additionally, dynamics of reference power is faster than the measured power, so the total energy control would response to changes faster. A low-pass filter is needed in order to remove the high frequency ripples on $V_{t}^{d c}$.

The filter time constant, must be designed adequately. The high-frequency resonances caused by the HVDC cable parameters can appear on $V_{t}^{d c}$. The frequency of resonance can range within a few hundreds of Hz. Therefore, the time constant should be selected to attenuate the resonance. In addition, it should not be larger than the time constant of power control loop, in order to avoid interactions between control loops.

\section{SYSTEM MODELING}

In this section, an overall linear model of the system based on the state-space equations is derived. The linear model contains the dynamics of both AC and DC networks.

\section{A. MMC Electrical Circuit}

Referring to Fig. 2, the electrical circuit equations per phase $(j=a, b, c)$ are

$$
\begin{aligned}
V_{u}^{\mathrm{dc}}-v_{u}^{j}-v_{g}^{j}-v_{n}= & R_{a} i_{u}^{j}+L_{a} \frac{d i_{u}^{j}}{d t}+\left(R_{s}+R_{g}\right) i_{s}^{j} \\
& +\left(L_{s}+L_{g}\right) \frac{d i_{s}^{j}}{d t} \\
-V_{l}^{\mathrm{dc}}+v_{l}^{j}-v_{g}^{j}-v_{n}= & -R_{a} i_{l}^{j}-L_{a} \frac{d i_{l}^{j}}{d t}+\left(R_{s}+R_{g}\right) i_{s}^{j} \\
& +\left(L_{s}+L_{g}\right) \frac{d i_{s}^{j}}{d t}
\end{aligned}
$$

where

- $R_{a}, L_{a}$ : arm resistance and inductance

- $R_{s}, L_{s}$ : coupling resistance and inductance (or transformer)

- $V_{u}^{D C}, V_{l}^{D C}$ : upper and lower voltages of the DC link

- $i_{s}^{j}, v_{g}^{j}$ : grid current and grid Thévenin voltage

- $v_{n}$ : grid voltage at the neutral point
- $v_{u}^{j}, v_{l}^{j}$ : voltages applied by the upper and lower arms

- $i_{u}^{j}, i_{l}^{j}$ : currents flowing through the upper and lower arms The following variable change is common in MMC modeling

$$
\left\{\begin{array} { l } 
{ v _ { \mathrm { diff } } ^ { j } \triangleq \frac { 1 } { 2 } ( - v _ { u } ^ { j } + v _ { l } ^ { j } ) } \\
{ v _ { \mathrm { sum } } ^ { j } \triangleq v _ { u } ^ { j } + v _ { l } ^ { j } } \\
{ i _ { \mathrm { sum } } ^ { j } \triangleq \frac { 1 } { 2 } ( i _ { u } ^ { j } + i _ { l } ^ { j } ) } \\
{ R \triangleq R _ { s } + R _ { g } + \frac { R _ { a } } { 2 } } \\
{ L \triangleq L _ { s } + L _ { g } + \frac { L _ { a } } { 2 } }
\end{array} \quad \left\{\begin{array}{l}
v_{u}^{j}=-v_{\mathrm{diff}}^{j}+\frac{1}{2} v_{\mathrm{sum}}^{j} \\
v_{l}^{j}=v_{\mathrm{diff}}^{j}+\frac{1}{2} v_{\mathrm{sum}}^{j} \\
i_{u}^{j}=\frac{1}{2} i_{s}^{j}+i_{\mathrm{sum}}^{j} \\
i_{l}^{j}=-\frac{1}{2} i_{s}^{j}+i_{\mathrm{sum}}^{j}
\end{array}\right.\right.
$$

where $v_{\text {diff }}^{j}, v_{\text {sum }}^{j}$, and $i_{\text {sum }}^{j}$, are, respectively, differential voltage, additive voltage, and additive current circulating from the upper to the lower arm of the leg $j$.

Adding and subtracting (4) and (5) and using the variable change given by (6) would lead to

$$
\begin{aligned}
& \frac{1}{2}\left(V_{u}^{\mathrm{dc}}-V_{l}^{\mathrm{dc}}\right)+v_{\mathrm{diff}}^{j}-v_{g}^{j}-v_{n}=R i_{s}^{j}+L \frac{d i_{s}^{j}}{d t} \\
& v_{\mathrm{sum}}^{j}-\left(V_{u}^{\mathrm{dc}}+V_{l}^{\mathrm{dc}}\right)=-2 R_{a} i_{\mathrm{sum}}^{j}-2 L_{a} \frac{d i_{\mathrm{sum}}^{j}}{d t}
\end{aligned}
$$

Equations (7) and (8) are decoupled and only contain a single derivative term $\left(i_{s}^{j}\right.$ and $\left.i_{\text {sum }}^{j}\right)$; hence, they are suitable for state-space representation. The current $i_{\text {sum }}^{j}$ contains AC and DC terms which impact the performance of the control system. Transforming the DC term into $\alpha \beta$-frame; the $\alpha \beta$ components regulate the internal power exchange between legs, while the zero component is used for the control of the power flow into the DC grid. In addition, the AC term contains $(+-0)$ sequences; the zero sequence is controlled to zero avoiding the $\mathrm{AC}$ current flow through DC grid, whereas $(+-)$ sequences can be used to regulate the internal power exchange between upper and lower arms of each leg. A comprehensive discussion can be found in [19].

Since the $(+-0)$ sequences of the $\mathrm{AC}$ term are relevant for internal energy balance studies [21], they can be ignored and only $\alpha \beta 0$ considered. Using the following definitions

$$
V_{\text {off }}^{\mathrm{dc}} \triangleq \frac{1}{2}\left(V_{u}^{\mathrm{dc}}-V_{l}^{\mathrm{dc}}\right), \quad V_{t}^{\mathrm{dc}} \triangleq V_{u}^{\mathrm{dc}}+V_{l}^{\mathrm{dc}}
$$

combined with (7) and (8), yields

$$
v_{\mathrm{diff}}^{a b c}-v_{g}^{a b c}+\left(V_{\mathrm{OFF}}^{\mathrm{dc}}-v_{n}\right)(111)^{T}=R \mathcal{I}_{\mathbf{3}} i_{s}^{a b c}+L \mathcal{I}_{\mathbf{3}} \frac{d i_{s}^{a b c}}{d t}
$$

$$
v_{\mathrm{sum}}^{\alpha \beta 0 \mathrm{dc}}-V_{t}^{\mathrm{dc}}\left(\begin{array}{llll}
0 & 0 & 1
\end{array}\right)^{T}=-2 R_{a} \mathcal{I}_{\mathbf{3}} i_{\mathrm{sum}}^{\alpha \beta 0 \mathrm{dc}}-2 L_{a} \mathcal{I}_{\mathbf{3}} \frac{d i_{\mathrm{sum}}^{\alpha \beta 0 \mathrm{dc}}}{d t}
$$

where $V_{\text {off }}^{\text {dc }}$ is half the imbalance between the voltages of the positive and negative HVDC poles, which is almost zero in normal operation. $\mathcal{I}_{\mathbf{3}}$ is an identity matrix of order 3.

\section{B. MTDC Grid}

The exemplary MTDC grid (Fig. 1) consists of several cable sections which can be modelled via state-space equations. Each 


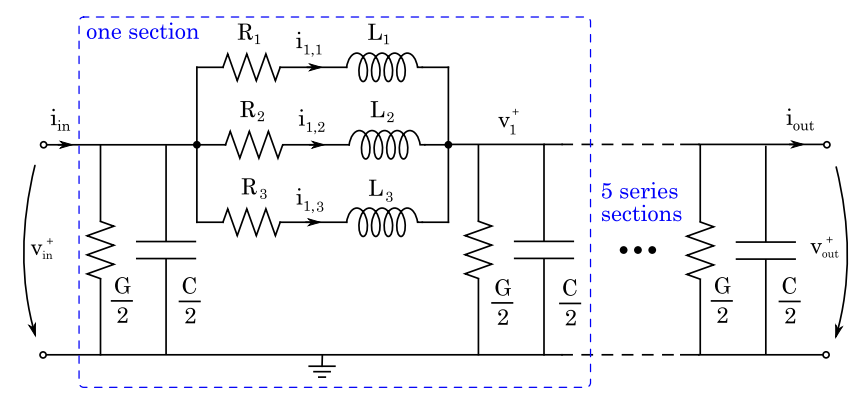

Fig. 8. HVDC cable model based on the lumped parameters vector fitting modelling approach.

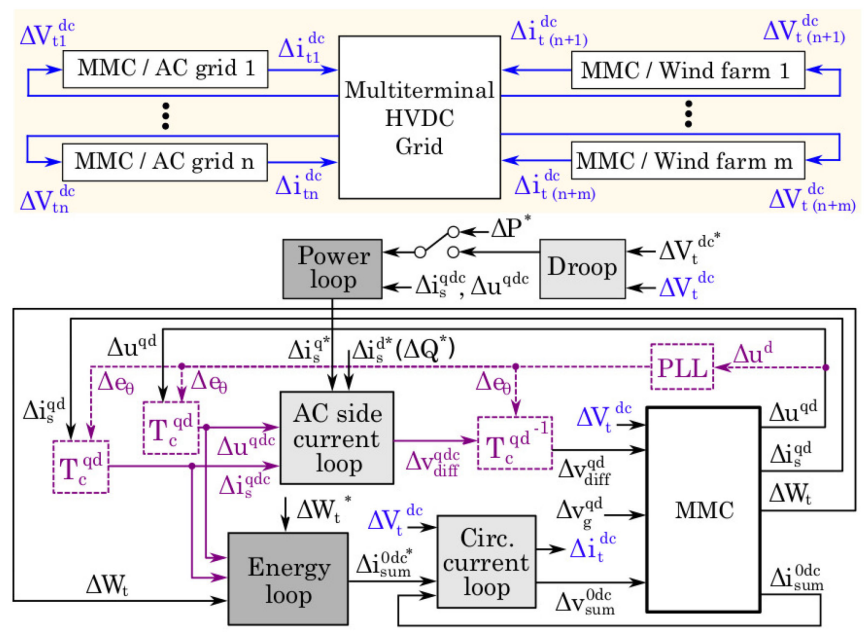

Fig. 9. Linear model of VSC-MTDC grid.

cable section is modeled using a lumped parameters vector fitting method which is developed in [22]. This modeling method utilizes several parallel RL branches to accurately reproduce cable electromagnetic transient behaviour, and more importantly, it is suitable for state-space representation. The cable model used in this study is shown in Fig. 8, which consists of five series subsections to account for the hyperbolic correction factors, improving cable model for long distances.

\section{System Overall Linear Model}

The overall linear model of an exemplary VSC-MTDC grid is obtained by expressing all control loops and circuit equations in linear state-space form, and interconnecting the state-space equations based on the similar inputs/outputs. As presented in Fig. 9, the state-space model of MTDC grid has the DC currents of MMCs as the inputs and provides DC voltages as the output. The overall linear state-space model of the VSC-MTDC grid is given by

$$
\Delta \dot{x}=\mathbf{A} \Delta x+\mathbf{B} \Delta u, \quad \Delta y=\mathbf{C} \Delta x+\mathbf{D} \Delta u
$$

where $\Delta \boldsymbol{x}$ is the state vector that includes states from the MMCs and the MTDC grid, $\Delta \boldsymbol{u}$ is the input vector containing control references and grid voltages, whereas $\Delta y$ is the output vector including controlled variables.

\section{Droop Gain Selection Methodology}

Once the linear model of the system is obtained, the droop gains can be selected based on the system steady-state and dynamic performances. The first step is to eliminate those droop gains that lead to the violation of DC voltage steady-state values from the limits. Then, the system dynamic response is evaluated for the remaining droop gains, leading to the final selection of the most suitable droop gains.

\section{A. System Steady-State Performance}

In the steady-state analysis, all MMCs are replaced by the voltage-controlled current sources and their internal dynamics are ignored. Referring to the exemplary VSC-MTDC system given in Fig. 1, the DC voltages at the terminals of all MMCs are calculated as

$$
\left[\begin{array}{c}
V_{t 1}^{\mathrm{dc}} \\
\vdots \\
V_{t n}^{\mathrm{dc}} \\
V_{t(n+1)}^{\mathrm{dc}} \\
\vdots \\
V_{t(n+m)}^{\mathrm{dc}}
\end{array}\right]=Z_{D C}\left[\begin{array}{c}
I_{t 1}^{\mathrm{dc}} \\
\vdots \\
I_{t n}^{\mathrm{dc}} \\
I_{t(n+1)}^{\mathrm{dc}} \\
\vdots \\
I_{t(n+m)}^{\mathrm{dc}}
\end{array}\right]
$$

where the vector of outputs contains $(n+m)$ DC voltages related to $n$ MMCs that control DC voltage, and $m$ MMCs which regulate power flow. $Z_{D C}$ is an MTDC grid impedance matrix. The DC currents of the MMCs are considered as the inputs, which can be expressed by the DC voltage droop equation and power reference

$$
\begin{aligned}
I_{t j}^{\mathrm{dc}}=\frac{\left(V_{t j}^{\mathrm{dc} *}-V_{t j}^{\mathrm{dc}}\right) k_{d j}}{V_{t j}^{\mathrm{dc}}} & j=1, \ldots, n \\
I_{t j}^{\mathrm{dc}}=\frac{P_{j}^{*}}{V_{t j}^{\mathrm{dc}}} & j=n+1, \ldots, n+m
\end{aligned}
$$

Substituting DC currents into (13), a set of nonlinear equations are derived which can be numerically solved for DC voltage values. The power and DC voltage references are considered as constant. The droop gains $\left(k_{d j}\right)$ that satisfy the steady-state performance of the DC voltages would be considered for the system dynamic evaluation.

\section{B. System Dynamic Performance}

Even if the steady-state values of DC voltages remain within the permissible range, the values of the system parameters may exceed the limits during transient. For any combination of droop gains applied to MMCs with droop control, the following system parameters (in per unit) should be studied, making sure that the VSC-MTDC grid operates within the permissible range:

- $\alpha_{p}$ (p.u.): permissible deviation of MMC power from ideal power sharing

- $\alpha_{v}$ (p.u.): permissible deviation of DC voltage, measured at any node of VSC-MTDC, from nominal value 
- $\alpha_{w}$ (p.u.): permissible deviation of MMC energy from the nominal value

- $\alpha_{q}$ (p.u.): AC current limitation of MMC

- $\alpha_{\text {sum }}$ (p.u.): DC current limitation of MMC

The following procedure is suggested to ensure that the selection of the droop gains would lead to the desired system dynamic performance:

1) Applying the droop combinations to the linear model, the transient power of MMCs should not exceed more than $\alpha_{p}$ from ideal power sharing. The droop combinations with undesirable results would be rejected.

2) The permissible droop combinations from the previous stage are studied for the DC voltage performance. The transient DC voltages of all MMCs (operating in DC voltage control and power control modes), should not deviate more than $\alpha_{v}$ from the nominal value. The droop combinations fail to meet criteria would be eliminated.

3) Once the droop combinations fulfilling both transient power sharing and DC voltage performance criteria are obtained, the AC and DC currents limitations of MMCs are investigated. The AC and additive (DC) currents of MMC, respectively, $i_{s}^{q}$ and $i_{\mathrm{sum}}^{\mathrm{Odc}}$, should not exceed the nominal values by more than, respectively, $\alpha_{q}$ and $\alpha_{\text {sum }}$.

4) Next, it is important to make sure that total energy also remains within the $\alpha_{w}$ deviation from its nominal value.

5) The small-signal stability analysis is conducted for all droop combinations, making sure none of them reducing the overall stability margin of the system.

6) Finally, once droop combinations considering all the above mentioned criteria are found, time-domain analysis is conducted on the non-linear model to validate results from linear analysis.

In order to study the dynamic performance of the above mentioned system parameters, the maximum singular values of various subsystems are studied. From the system overall small-signal model given in Fig. 9, the following subsystems are of interest for droop design purpose:

$$
\begin{aligned}
\boldsymbol{Y}_{\boldsymbol{p}} & =\boldsymbol{G}_{\boldsymbol{p}}(s) \boldsymbol{U}, & \boldsymbol{Y}_{\boldsymbol{p}} & =\left[\Delta P_{1}, \ldots, \Delta P_{n}\right]^{T} \\
\boldsymbol{Y}_{\boldsymbol{v}} & =\boldsymbol{G}_{\boldsymbol{v}}(s) \boldsymbol{U}, & \boldsymbol{Y}_{\boldsymbol{v}} & =\left[\Delta V_{t 1}^{d c}, \ldots, \Delta V_{t(n+m)}^{d c}\right]^{T} \\
\boldsymbol{Y}_{\boldsymbol{w}} & =\boldsymbol{G}_{\boldsymbol{w}}(s) \boldsymbol{U}, & \boldsymbol{Y}_{\boldsymbol{w}} & =\left[\Delta W_{t 1}, \ldots, \Delta W_{t n}\right]^{T} \\
\boldsymbol{Y}_{\boldsymbol{q}} & =\boldsymbol{G}_{\boldsymbol{q}}(s) \boldsymbol{U}, & \boldsymbol{Y}_{\boldsymbol{q}} & =\left[\Delta i_{s 1}^{q}, \ldots, \Delta i_{s n}^{q}\right]^{T} \\
\boldsymbol{Y}_{\text {sum }} & =\boldsymbol{G}_{\text {sum }}(s) \boldsymbol{U}, & \boldsymbol{Y}_{\text {sum }} & =\left[\Delta i_{\text {sum } 1}^{0 d c}, \ldots, \Delta i_{\text {sumn }}^{0 d c}\right]^{T}
\end{aligned}
$$

where, $n$ and $m$ are the number of, respectively, the MMCs regulating DC voltage via droop and the MMCs operating in power control mode. $\boldsymbol{U}$ is a vector of power set points $\left[\Delta P_{(n+1)}, \ldots, \Delta P_{(n+m)}\right]$. Applying singular value analysis, the maximum singular values of $G_{p}(s)$ should not exceed the following gains:

$$
\bar{\sigma}\left(G_{p}(s)\right) \leq \frac{\left\|y_{p}(0)\right\|_{2}}{\|u(0)\|_{2}}
$$

being $u(0)$ the input containing power set points and $y_{p}(0)$ the corresponding output. This analysis is also conducted on DC

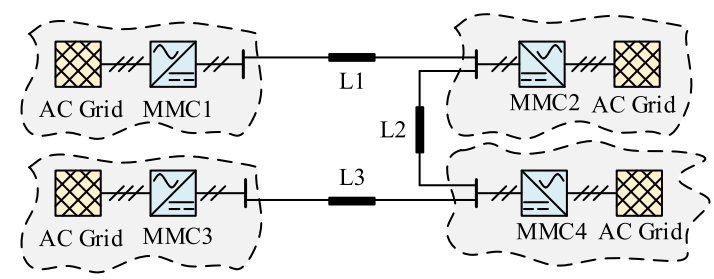

Fig. 10. Case study: a four-terminal HVDC Grid.

TABLE I

MMC AND AC GRID PARAMETERS

\begin{tabular}{lccc}
\hline Parameter & Symbol & Value & Units \\
\hline Rated (base) active power & $P_{N}$ & 500 & $\mathrm{MW}$ \\
Rated (base) ac-side voltage & $U_{N}$ & 320 & $\mathrm{kV} \mathrm{rms} \mathrm{ph-ph}$ \\
Rated (base) dc-side voltage & $V_{\mathrm{dc}}$ & \pm 320 & $\mathrm{kV}$ \\
Grid short-circuit ratio & $\mathrm{SCR}$ & 10 & - \\
Coupling impedance & $R_{s}+\mathrm{j} L_{s}$ & $0.01+\mathrm{j} 0.2$ & $\mathrm{pu}$ \\
Arm reactor impedance & $R_{a}+\mathrm{j} L_{a}$ & $0.01+\mathrm{j} 0.2$ & $\mathrm{pu}$ \\
Converter modules per arm & $N_{\mathrm{arm}}$ & 400 & - \\
Average module voltage & $V_{\mathrm{SM}}$ & 1.6 & $\mathrm{kV}$ \\
Sub-module capacitance & $C_{\mathrm{SM}}$ & 8 & $\mathrm{mF}$ \\
\hline
\end{tabular}

TABLE II

HVDC CABLE PARAMETERS

\begin{tabular}{lccccc}
\hline Symbol & Value & Units & Symbol & Value & Units \\
\hline$r_{1}$ & 0.1265 & $\Omega / \mathrm{km}$ & $l_{1}$ & 0.2644 & $\mathrm{mH} / \mathrm{km}$ \\
$r_{2}$ & 0.1504 & $\Omega / \mathrm{km}$ & $l_{2}$ & 7.2865 & $\mathrm{mH} / \mathrm{km}$ \\
$r_{3}$ & 0.0178 & $\Omega / \mathrm{km}$ & $l_{3}$ & 3.6198 & $\mathrm{mH} / \mathrm{km}$ \\
$c$ & 0.1616 & $\mu \mathrm{F} / \mathrm{km}$ & $g$ & 0.1015 & $\mu \mathrm{S} / \mathrm{km}$ \\
\hline
\end{tabular}

voltage and total energy transfer function matrices, $G_{v}(s)$ and $G_{w}(s)$, to ensure the transient values do not exceed the limits. Moreover, the MMCs current limitations are studied via $G_{q}(s)$ and $G_{\text {sum }}(s)$. The following limits are applied to maximum singular values:

$$
\begin{aligned}
y_{p}(0) & =\left(1+\alpha_{p}\right) \times \frac{1}{m} \sum_{j=n+1}^{n+m} P_{j}^{*} \\
y_{v}(0) & =\alpha_{v} V_{t}^{d c *},(\max . \text { DC voltage deviation }) \\
y_{w}(0) & =\alpha_{w} W_{t}^{*},(\text { max. energy deviation }) \\
y_{q}(0) & =\left(1+\alpha_{q}\right) P_{N} /\left(3 / 2 U_{N}\right),(\max . \text { AC current }) \\
y_{\text {sum }}(0) & =\left(1+\alpha_{\text {sum }}\right) P_{N} /\left(3 V_{t}^{d c *}\right),(\text { max. DC current })
\end{aligned}
$$

\section{EVALUATION OF Droop CONTROL SyStem}

In this section, two studies are conducted: first, the performance of the droop selection methodology is tested on the fourterminal DC grid shown in Fig. 10, and second, the performances of the classic and improved cross control approaches for DC voltage regulation via droop controllers are compared. Referring to Fig. 10, MMC 1 and MMC 3 have improved cross control structure and regulate DC voltage with droop controllers, while MMC 2 and MMC 4 have classic control structure and operate in power control mode. It is assumed that cable $\mathrm{L} 2$ is $80 \mathrm{~km}$ long and connected between MMC 2 and MMC 4 terminals, and L1 and L3 are $350 \mathrm{~km}$ and $200 \mathrm{~km}$, respectively. The cable and system parameters are given in Tables I and II. 

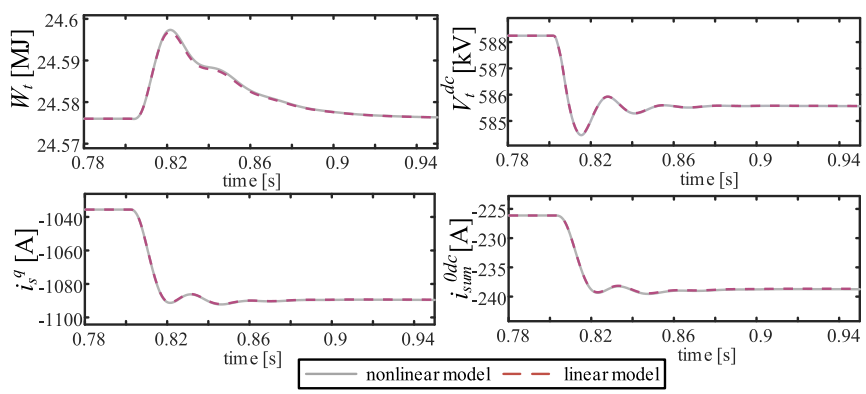

Fig. 11. Comparison of the nonlinear and linear system responses. Only variables of MMC 1 are presented.

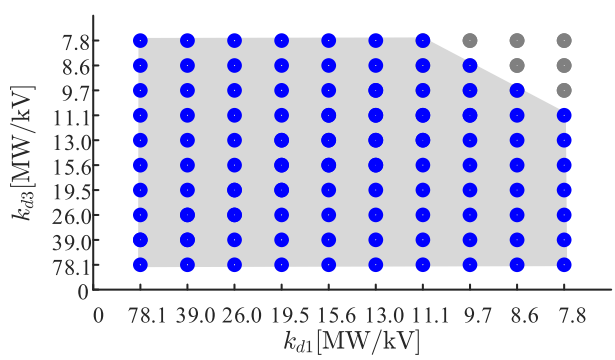

Fig. 12. Permissible droop combination (blue circles) based on the steady-state values of DC voltages. Six droop combinations lead to the DC voltage deviations more than $10 \%$ of nominal value.

The voltage deviation, $\Delta V_{t}^{d c}$, is in percentage within the range of 1 to 10 percent of $V_{t}^{d c *}$. Changing voltage deviation in steps of one percent, $k_{d}$ can be calculated from (3), which can be written as

$$
k_{d}=\frac{P_{t}^{*}}{\Delta V_{t}^{d c} \times V_{t}^{d c *}}
$$

The droop gains would be within the range of 7.81 to $78.12 \mathrm{MW} / \mathrm{kV}$ :

$$
k_{d}=[78.1,39.0,26.0,19.5,15.6,13.0,11.1,9.7,8.6,7.8]
$$

\section{A. Linear Model Validation}

The validation of the system linear model (Fig. 9) is carried out in Matlab Simulink. Assuming the power injected into the system by MMC 2 and $4\left(P_{2}\right.$ and $\left.P_{4}\right)$ is increased by $5 \%$ each (10\% in total), the responses of the nonlinear and linear models are compared in Fig. 11, which indicates a good accuracy.

\section{B. Droop Gain Selection}

While depending on the VSC-MTDC grid, any limitation can be applied to the analysis, the following reasonable limits are considered: $\alpha_{p}=20 \%, \alpha_{v}=10 \%, \alpha_{w}=10 \%, \alpha_{q}=20 \%$, $\alpha_{\text {sum }}=20 \%$.

The droop gains are selected from vector $\boldsymbol{k}_{\boldsymbol{d}}$. Therefore, $k_{d 1}$ and $k_{d 3}$ can have 100 combinations that should be studied to find the ones fulfilling all criteria. Using (13), the steady-state analysis reveals that from 100 droop combinations, 6 droop combinations would cause the steady-state values of DC voltages to violate the limits (see Fig. 12). Note that, although MMC 1 and
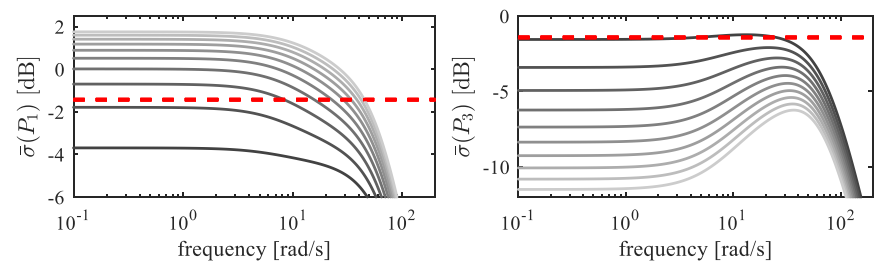

Fig. 13. Maximum singular values of $P_{1}$ and $P_{3}$ for ten values of $k_{d 3}$, while $k_{d 1}$ is fixed at $78.1 \mathrm{MW} / \mathrm{kV}$.

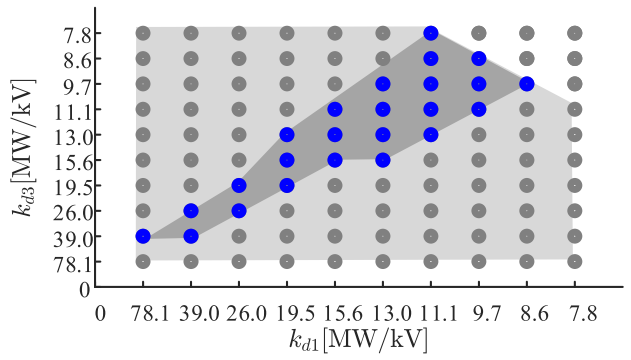

Fig. 14. Permissible droop combination (blue circles) for the maximum deviation of $20 \%$ from ideal power sharing.

3 maintain their DC voltages within the acceptable range, the DC voltages of MMC 2 and 4 would exceed the limits.

After identification of the droop combinations that fulfil DC voltage limits in steady-state conditions (94 droop combinations), the system dynamics are studied for these droop combinations.

1) Transient Power Sharing: Assuming $k_{d 1}$ is set at 78.1 $\mathrm{MW} / \mathrm{kV}$ and $k_{d 3}$ is adopting all values from $k_{d}$, the maximum singular values of $G_{p}(s)$ are presented in Fig. 13. The maximum singular values of $P_{1}$ exceed the limit (20\% of deviation from ideal transient power sharing) for a wide range of droop gains and remain within the allowable range for the droop combination $\left(k_{d 1}, k_{d 3}\right) \mathrm{MW} / \mathrm{kV}$ of $(78.1,78.1)$ and $(78.1,39.0)$. However, the singular values of $P_{3}$ exceed the limit for the combination (78.1,78.1). Hence, only the droop combination $(78.1,39.0)$ would be valid. Continuing this approach to investigate all 94 droop combinations, the following 24 droop combinations that are shown in Fig. 14 are valid since they satisfy transient power sharing criteria.

2) DC Voltage Performance: Among 24 droop combinations, only those satisfying DC voltage performance criteria are acceptable. If the maximum singular values related to each row of $G_{v}(s)$ remain below the limit, it can be ensured that DC voltages never violate a maximum $10 \%$ deviation from nominal value.

The maximum singular values of $G_{v}(s)$ are shown in Fig. 15. The droop combinations are divided into three groups of 8 droop combinations for the ease of presentation. For the droop combinations 1 to 8 , none of DC voltages violates the limit, whereas, all droop combinations 17 to 24 violate the limit.

Note that, although all droop combinations are calculated for a maximum voltage deviation of $10 \%$, the $V_{t 2}^{d c}$ and $V_{t 4}^{d c}$ are not controlled via droop controllers, so they exhibit higher overshoot. Moreover, the droop combinations 1 to 8, which 

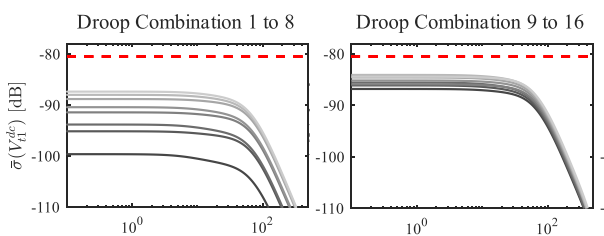

Droop Combination 17 to 24
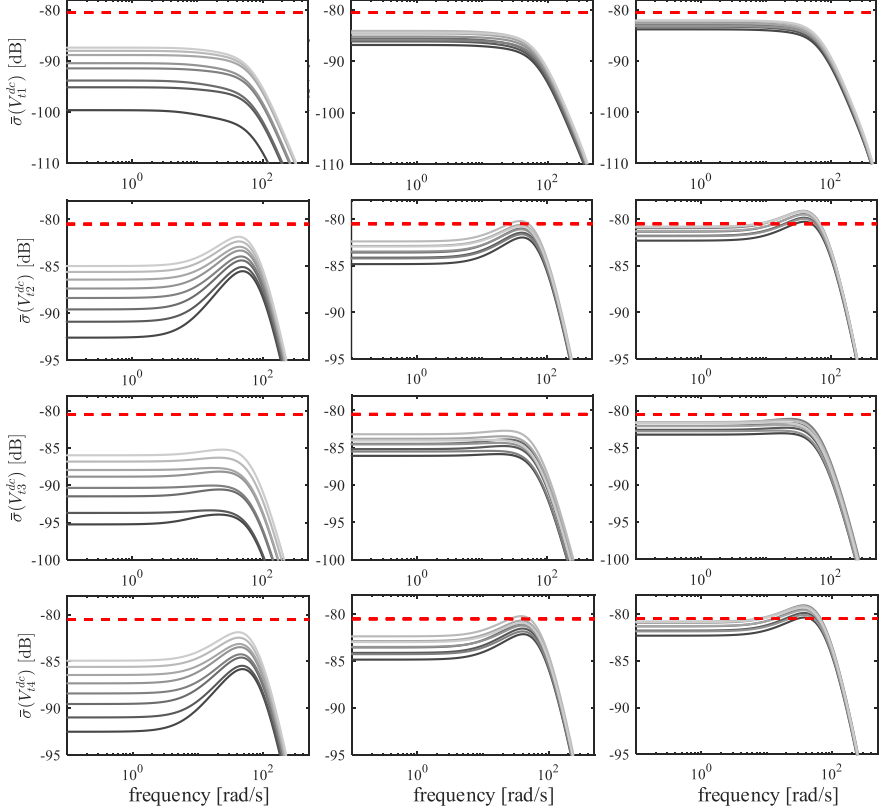

Fig. 15. Maximum singular values of $V_{t 1}^{d c}, V_{t 2}^{d c}, V_{t 3}^{d c}$, and $V_{t 4}^{d c}$ for three groups of droop combinations.

contain larger droop gains (see Fig. 14) are more suitable for a better DC voltage performance. Finally, 9 out of 24 droop combinations fail to fulfill DC voltage performance criteria.

3) Current Limitations: The AC and DC current limitations of MMCs are studied for 24 droop combinations. Assuming that MMC is able to withstand $20 \%$ of overcurrent on its DC and AC sides for a limited period of time, the maximum singular values of each row of $G_{q}(s)$ and $G_{\text {sum }}(s)$ should not exceed the limits defined earlier. As it is presented in Fig. 16, while $i_{s 1}^{q}$ is always below the limit, other currents exceed the limit for several droop combinations.

4) Total Energy Performance: The total energy has to be also within the permissible range as its violation may endangers converter performance. The total energy remains under the limit for all 24 droop combinations, which is presented in Fig. 17. Hence, total energy does not impose constraints on the droop gain selection.

5) Overall System Stability: It is also of importance to make sure that system overall stability margin does not reduce for various droop combinations. As it is presented in Fig. 18, for all 24 droop combinations, overall stability of the system is maintained.

After the elimination of the droop combinations which violate transient power sharing, DC voltage, or overcurrent limits, only 12 out of 24 droop combinations fulfil all the constraint, which are shown in Fig. 19. While the DC voltage steady-state analysis identifies 94 valid droop combinations for the system (Fig. 12), the dynamic performance analysis validates only 12 of them.

6) Time-Domain Validation: The final 12 droop combinations are validated with nonlinear model using time domain analysis. MMC 2 and MMC 4 reach to their nominal power
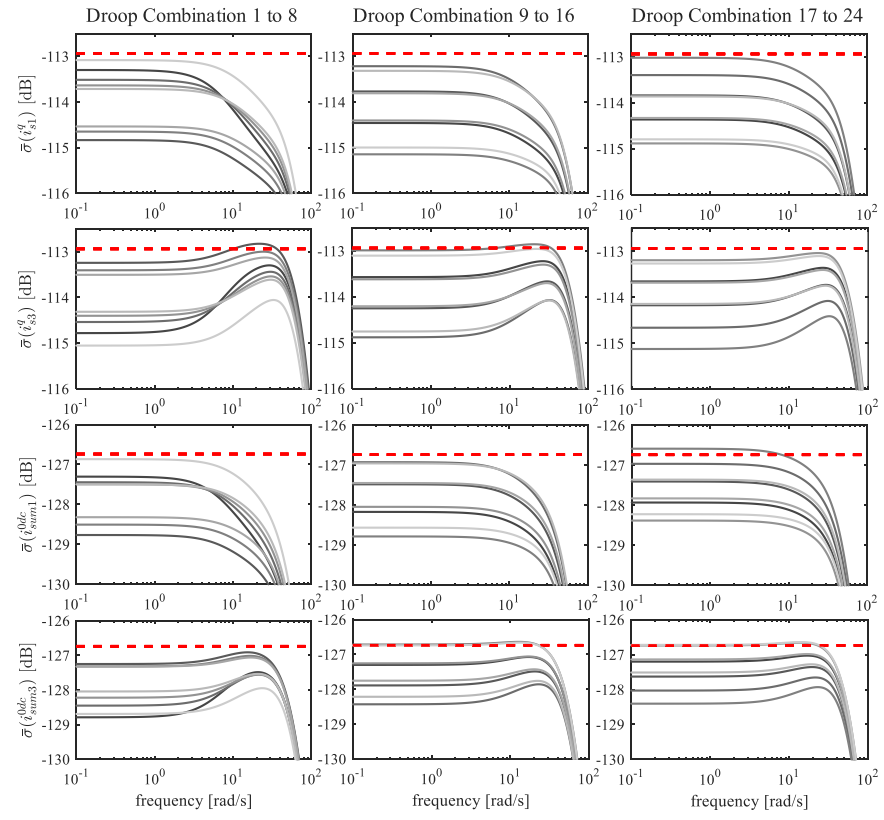

Fig. 16. Maximum singular values of $i_{s 1}^{q}, i_{s 3}^{q}, i_{s u m 1}^{0 d c}$, and $i_{s u m 3}^{0 d c}$ for three groups of droop combinations.
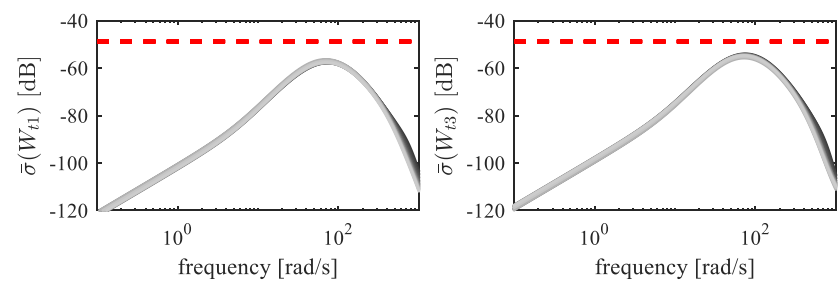

Fig. 17. Maximum singular values of $W_{t 1}$ and $W_{t 3}$ for all 24 droop combinations.

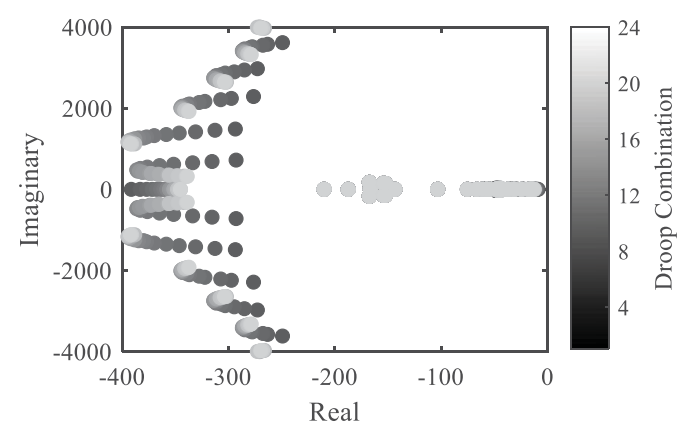

Fig. 18. System eigenvalues for 24 droop combinations.

at $0.15 \mathrm{~s}$. As it is indicated in Fig. 20, the generated DC power by MMC 1 and $3, P_{1}$ and $P_{3}$, do not exceed $20 \%$ from the ideal power sharing during transient. The steady-state deviation from ideal power sharing can be compensated by a secondary controller.

Next, the DC voltage performance and current dynamics are shown in, respectively, Fig. 21 and Fig. 22. For all 12 droop 


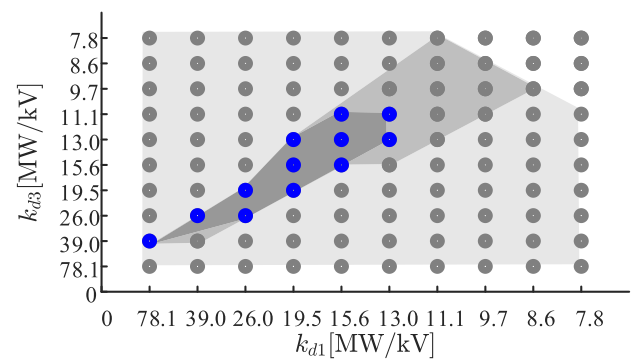

Fig. 19. Final 12 permissible droop combinations.
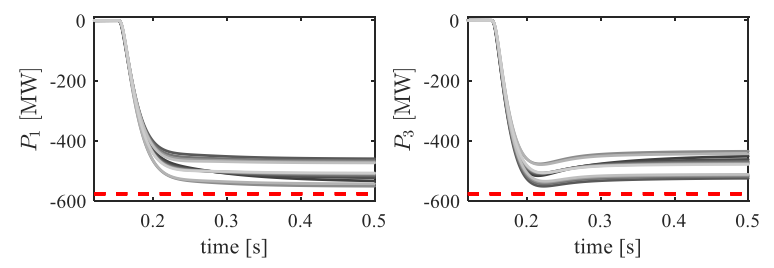

Fig. 20. The transient deviation of $P_{1}$ and $P_{3}$ from ideal power sharing for the final 12 droop combinations.
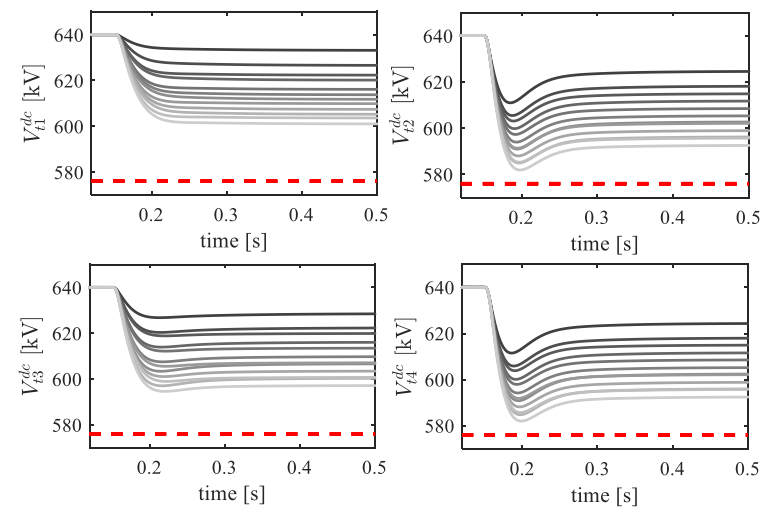

Fig. 21. Transient response of DC voltages for the final 12 droop combinations.

combinations, no DC voltage or current exceeds the limit, which is consistent with the linear analysis. Moreover, the total energy transient and steady-state response never exceed the limits. As presented in Fig. 23, $W_{t 1}$ and $W_{t 3}$ are far from the limit.

Since all final 12 droop combinations fulfil power sharing, voltage, energy, and current requirements, it might be of interest to select the droop combination with the lowest voltage deviation and highest power sharing accuracy in steady state. To do this, the 12 droop combinations are ranked for the voltage deviation from the lowest to highest as $[1,2,3,4,5,6,7,8,9,10,11,12]$, and for the power sharing from the highest accuracy to the lowest as $[12,9,11,6,8,4,5,3,2,1,10,7]$. It may be logical to choose droop combination 4 , with the droop gains $(26.0,19.5)$, which gives the maximum voltage deviation of $3 \%$ and $4 \%$ for $V_{t 1}^{d c}$ and $V_{t 3}^{d c}$, respectively, while it limits the power difference between $P_{1}$ and $P_{3}$ to $50 \mathrm{MW}$ at full load change.
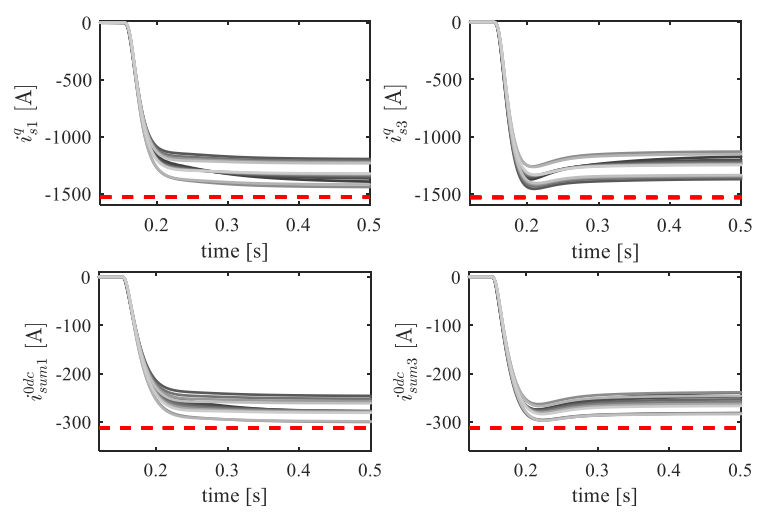

Fig. 22. Transient response of $i_{s}^{q}$ and $i_{\text {sum }}^{0 d c}$ for the final 12 droop combinations.
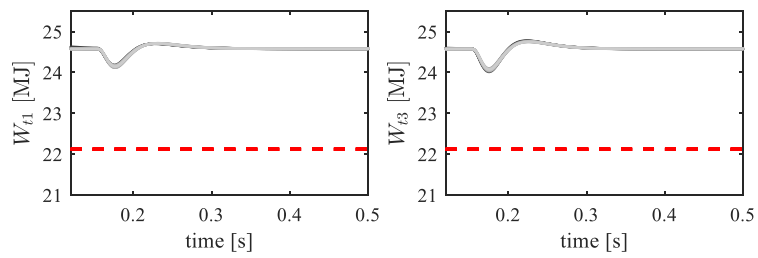

Fig. 23. Transient response of $W_{t 1}$ and $W_{t 3}$ for the final 12 droop combinations.

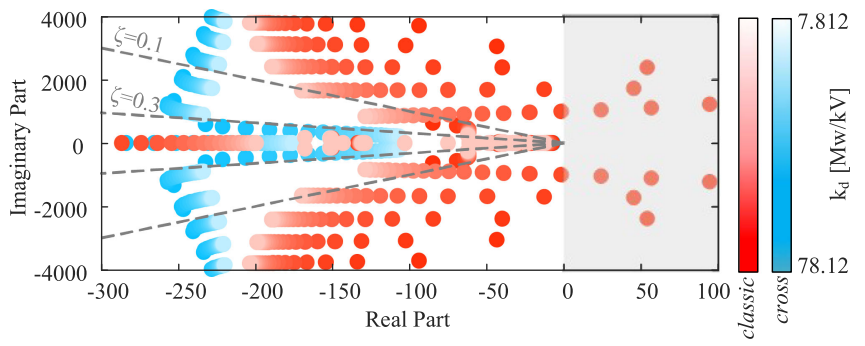

Fig. 24. Droop controller performance comparison; classic vs improved cross control approaches for various droop gains.

\section{Droop Performance Comparison Between Classic and Improved Cross Control Systems}

It is constructive to illustrate the dynamic behaviour of the improved cross control system against classic control in VSCMTDC grid. Referring to Fig. 10, the MMCs 2 and 4 operate in power mode control, so classic control systems are considered for them. Regarding to MMC 1 and 3, which regulate DC voltage via droop, they can have either improved cross or classic control. So the system dynamics and the performance of the droop gain selection methodology are compared for these two cases.

The system eigenvalues for the implementation of the improved cross and classic control systems on MMC 1 and 3 are presented in Fig. 24. As the droop gains increase (equivalent to $\Delta V_{t}^{d c}$ decrease), the eigenvalues of classic control approach move towards the instability region. However, the system with the improved cross control remains stable for all droop gains, revealing that the improved cross control is more suitable for the purpose of droop control design. 


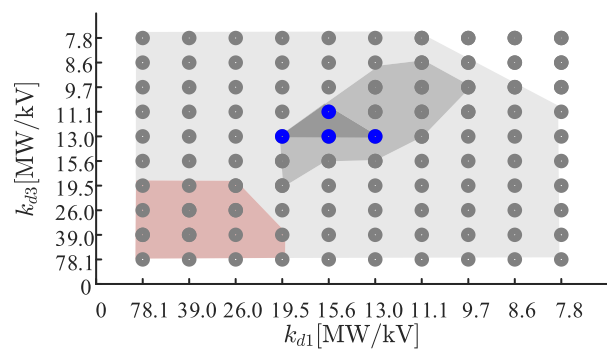

Fig. 25. Final 3 permissible droop combinations in case all MMCs have classic control systems. The red area is related to the unstable system.

Moreover, using the classic control for MMC 1 and 3 (i.e. all MMCs have classic control) and following the droop gain selection methodology, the final permissible droop combinations are presented in Fig. 25. While 12 droop combinations meet the system criteria using the improved cross control (see Fig. 19), only 4 droop combinations fulfil the criteria in case of classic control. However, in both cases, the transient power sharing and DC voltage dynamics significantly restrict the permissible droop gain combinations. Since the DC and AC current do not impose significant limits on the droop gain selection, it seems that the restrictions caused by transient power sharing are also due to the dynamics of the DC voltage. The total energy dynamics do not restrict the permissible droop gain combinations in classic and improved cross control systems; however, this statement is valid in case the reference for the total energy is constant and the main system disturbance is the power fluctuations. Finally, contrary to the improved cross control in which all droop gains lead to a stable system, the classic control is not stable for several droop gain combinations (the red area in Fig. 25).

\section{CONCLUSION}

In this paper, a detailed analysis and design of the DC voltage droop control for MMC-based MTDC grids were conducted. First, it has been concluded that the cross control structure is more adequate for DC voltage droop control. Inspired by the cross control, an improved MMC control strategy which is more robust against disturbances was derived. Then, a methodology was suggested to select droop gain combinations, considering the $\mathrm{AC}$ grid, $\mathrm{DC}$ grid and $\mathrm{MMC}$ dynamics and limitations. The proposed methodology was tested on a four-terminal HVDC grid. Initially, 100 droop gain combinations for the two MMCs regulating the DC voltage were calculated based on an acceptable steady-state voltage deviation. Then, following the suggested methodology, in every step a limitation was imposed on the maximum singular value representation, such as transient power sharing, DC voltage deviation, AC and DC currents, total energy deviation, also verifying the small signal stability for all droop combinations. From 100 initial droop combinations, only 12 droop combinations fulfilled all the criteria, which were tested on the nonlinear model using time-domain analysis to validate linear analysis results. The derived methodology is a comprehensive tool which ensures that neither the converters nor the system limits are exceeded when the MTDC grid is operating in droop control mode.

\section{REFERENCES}

[1] E. Prieto-Araujo, A. Egea-Alvarez, S. Fekriasl, and O. Gomis-Bellmunt, "DC voltage droop control design for multiterminal hvdc systems considering ac and dc grid dynamics," IEEE Trans. Power Del., vol. 31, no. 2, pp. 575-585, Apr. 2016.

[2] Q. Peng, T. Liu, S. Wang, Y. Qiu, X. Li, and B. Li, "Determination of droop control coefficient of multi-terminal VSC-HVDC with system stability consideration," IET Renewable Power Gener., vol. 12, no. 13, pp. 1508$1515,2018$.

[3] L. Zhang et al., "Modeling, control, and protection of modular multilevel converter-based multi-terminal hvdc systems: A review," CSEE J. Power Energy Syst., vol. 3, no. 4, pp. 340-352, Dec. 2017.

[4] W. Feng, L. A. Tuan, L. B. Tjernberg, A. Mannikoff, and A. Bergman, "A new approach for benefit evaluation of multiterminal VSC-HVDC using a proposed mixed ac/dc optimal power flow," IEEE Trans. Power Del., vol. 29, no. 1, pp. 432-443, Feb. 2014.

[5] N. Flourentzou, V. G. Agelidis, and G. D. Demetriades, "VSC-based HVDC power transmission systems: An overview," IEEE Trans. Power Electron., vol. 24, no. 3, pp. 592-602, Mar. 2009.

[6] A. Egea-Alvarez, F. Bianchi, A. Junyent-Ferre, G. Gross, and O. GomisBellmunt, "Voltage control of multiterminal VSC-HVDC transmission systems for offshore wind power plants: Design and implementation in a scaled platform," IEEE Trans. Ind. Electron., vol. 60, no. 6, pp. 2381-2391, Jun. 2013.

[7] J. Liang, T. Jing, O. Gomis-Bellmunt, J. Ekanayake, and N. Jenkins, "Operation and control of multiterminal HVDC transmission for offshore wind farms," IEEE Trans. Power Del., vol. 26, no. 4, pp. 2596-2604, Oct. 2011.

[8] F. Thams, R. Eriksson, and M. Molinas, "Interaction of droop control structures and its inherent effect on the power transfer limits in multiterminal VSC-HVDC," IEEE Trans. Power Del., vol. 32, no. 1, pp. 182-192, Feb. 2017

[9] W. Wang, M. Barnes, and O. Marjanovic, "Stability limitation and analytical evaluation of voltage droop controllers for VSC MTDC," CSEE J. Power Energy Syst., vol. 4, no. 2, pp. 238-249, Jun. 2018.

[10] J. Freytes et al., "Dynamic analysis of MMC-based MTDC grids: Use of MMC energy to improve voltage behavior," IEEE Trans. Power Del., vol. 34, no. 1, pp. 137-148, Feb. 2019.

[11] E. Sánchez-Sánchez, E. Prieto-Araujo, and O. Gomis-Bellmunt, "Multiterminal HVDC voltage droop control design considering dc grid, ac grid and MMC dynamics," in Proc. 13th IET Int. Conf. AC DC Power Transmiss., Feb. 2017, pp. 1-6.

[12] P. Wang, X. Zhang, P. F. Coventry, and R. Zhang, "Start-up control of an offshore integrated MMC multi-terminal HVDC system with reduced dc voltage," IEEE Trans. Power Syst., vol. 31, no. 4, pp. 2740-2751, Jul. 2016.

[13] W. Wang, A. Beddard, M. Barnes, and O. Marjanovic, "Analysis of active power control for VSC HVDC," IEEE Trans. Power Del., vol. 29, no. 4, pp. 1978-1988, Aug. 2014.

[14] C. Alcota and M. A. Perez, "Control of a multi-terminal de transmission system based on local variables," in Proc. IEEE Int. Conf. Ind. Technol. (ICIT), Mar. 2016, pp. 482-486.

[15] M. M. Belhaouane et al., "Optimal control design for modular multilevel converters operating on multi-terminal dc grid," in Proc. Power Syst. Comput. Conf., Jun. 2016, pp. 1-7.

[16] N. Yousefpoor and S. Bhattacharya, "Control and dynamic performance evaluation of multi-terminal dc grid," in Proc. IEEE Power Energy Soc. General Meet., Jul. 2015, pp. 1-5.

[17] T. M. Haileselassie and K. Uhlen, "Impact of dc line voltage drops on power flow of MTDC using droop control," IEEE Trans. Power Syst., vol. 27, no. 3, pp. 1441-1449, Aug. 2012.

[18] O. Gomis-Bellmunt, J. Liang, J. Ekanayake, and N. Jenkins, "Voltagecurrent characteristics of multiterminal HVDC-VSC for offshore wind farms," Electric Power Syst. Res., vol. 81, no. 2, pp. 440-450, 2011. [Online]. Available: http://www.sciencedirect.com/science/article/ pii/S0378779610002403

[19] E. Prieto-Araujo, A. Junyent-Ferré, C. Collados-Rodríguez, G. ClarianaColet, and O. Gomis-Bellmunt, "Control design of modular multilevel converters in normal and ac fault conditions for HVDC grids," Electric Power Syst. Res., vol. 152, pp. 424-437, 2017.

[20] E. Sánchez-Sánchez, E. Prieto-Araujo, A. Junyent-Ferré, and O. GomisBellmunt, "Analysis of mmc energy-based control structures for VSCHVDC links," IEEE J. Emerg. Sel. Topics Power Electron., vol. 6, no. 3, pp. 1065-1076, Sep. 2018. 
[21] A. Junyent-Ferré, P. Clemow, M. M. C. Merlin, and T. C. Green, "Operation of HVDC modular multilevel converters under dc pole imbalances," in Proc. 16th Eur. Conf. Power Electron. Appl., Aug. 2014, pp. $1-10$.

[22] J. Beerten, S. D'Arco, and J. A. Suul, "Frequency-dependent cable modelling for small-signal stability analysis of VSC-HVDC systems," IET Gener, Transmiss. Distribution, vol. 10, no. 6, pp. 1370-1381, 2016.

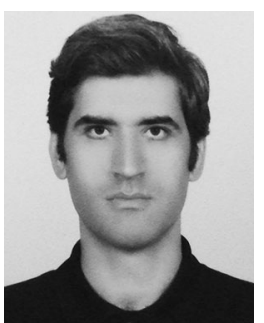

Saman Dadjo Tavakoli received the M.S. degree in electrical engineering from Shahid Beheshti University, Tehran, Iran, in 2015 . He is currently working toward the Ph.D. degree in electrical engineering as an early-stage Researcher as part of InnoDC (Innovative tools for offshore wind and dc grids) project with the Technical University of Catalonia (UPC), Barcelona, Spain. His research interests include multi-terminal HVdc systems, dc microgrids, power system stability, and advanced control system design.

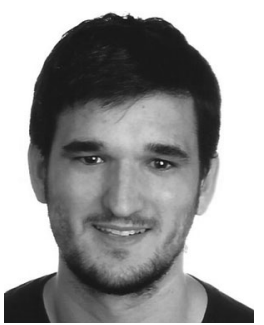

Enric Sánchez-Sánchez (Student Member, IEEE) received the degree in industrial engineering from the School of Industrial Engineering of Barcelona, Technical University of Catalonia (UPC), Barcelona, Spain, in 2014, where he is currently working toward the Ph.D. degree in electrical engineering. In 2012, he joined CITCEA-UPC as an Intern, where he became a Project Engineer in 2014. His research interests include modeling and control of power electronics converters integrated into the power system, HVdc transmission, and renewable generation systems.

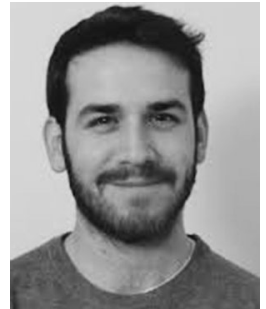

Eduardo Prieto-Araujo (Member, IEEE) received the degree in industrial engineering from the School of Industrial Engineering of Barcelona, Technical University of Catalonia (UPC), Barcelona, Spain, in 2011 and the Ph.D. degree in electrical engineering from the UPC in 2016. He joined CITCEA-UPC research group in 2010 and currently he is a Serra Húnter lecturer with the Electrical Engineering Department, UPC. His main interests are renewable generation systems, control of power converters for $\mathrm{HVdc}$ applications, interaction analysis between converters, and power electronics dominated power systems.

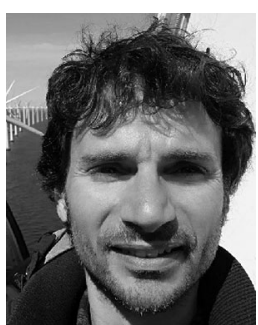

Oriol Gomis-Bellmunt (Senior Member, IEEE) received the degree in industrial engineering from the School of Industrial Engineering of Barcelona, Technical University of Catalonia (UPC), Barcelona, Spain, in 2001 and the Ph.D. degree in electrical engineering from the UPC in 2007. In 1999, he joined Engitrol S.L. where he worked as Project Engineer in the automation and control industry. Since 2004, he has been with the Electrical Engineering Department, UPC where he is a Professor and participates in the CITCEA-UPC Research Group. He is currently an ICREA Academia researcher. His research interests include the fields linked with electrical machines, power electronics, and renewable energy integration in power systems. 